\title{
Tolerating Byzantine Faulty Clients in a Quorum System
}

\author{
Barbara Liskov \\ MIT CSAIL \\ Cambridge, MA, USA
}

\author{
Rodrigo Rodrigues \\ INESC-ID / Instituto Superior Técnico \\ Lisbon, Portugal
}

\begin{abstract}
Byzantine quorum systems have been proposed that work properly even when up to $f$ replicas fail arbitrarily. However, these systems are not so successful when confronted with Byzantine faulty clients. This paper presents novel protocols that provide atomic semantics despite Byzantine clients. Our protocols prevent Byzantine clients from interfering with good clients: bad clients cannot prevent good clients from completing reads and writes, and they cannot cause good clients to see inconsistencies. In addition we also prevent bad clients that have been removed from operation from leaving behind more than a bounded number of writes that could be done on their behalf by a colluder.

Our protocols are designed to work in an asynchronous system like the Internet and they are highly efficient. We require $3 f+1$ replicas, and either two or three phases to do writes; reads normally complete in one phase and require no more than two phases, no matter what the bad clients are doing.

We also present strong correctness conditions for systems with Byzantine clients that limit what can be done on behalf of bad clients once they leave the system. Furthermore we prove that our protocols are both safe (they meet those conditions) and live.
\end{abstract}

\section{Introduction}

Quorum systems [4, 13] are valuable tools for building highly available replicated data services. A quorum system can be defined as a set of sets (called quorums) with certain intersection properties. These systems allow read and write operations to be performed only at a quorum of the servers, since the intersection properties ensure that any read operation will have access to the most recent value that was written.

The original work on quorum systems assumed that servers fail benignly, i.e., by crashing or omitting some steps. More recently, researchers have developed techniques that enable quorum systems to provide data availability even in the presence of arbitrary (Byzantine) faults [9]. Earlier work provides correct semantics despite server (i.e., replica) failures and also handles some of the problems of Byzantine clients [2, 3, 5, 9, 10, 11, 12].

This paper extends this earlier work in two important ways. First, it defines new protocols that efficiently handle more problems caused by Byzantine clients than previous approaches. Our protocols compare favorably to all previous proposals: they either rely on weaker assumptions (e.g., about the network), or they are more efficient in terms of operation latency and number of replicas.

Second, the paper formally defines novel correctness conditions for Byzantine quorum systems, and proves that our protocols meet such conditions. The correctness conditions are stronger than what has been stated previously [11] and what has been guaranteed by previous approaches.

Since a dishonest client can write garbage into the shared variable, it may seem there is little value in limiting what bad clients can do. But this is not the case, for two reasons. First, bad clients can cause a protocol to misbehave so that good clients are unable to perform operations (i.e., the protocol is no longer live) or observe incorrect behavior. For example, if the variable is write-once, a good client might observe that its state changes multiple times.

Second, bad clients can continue to interfere with good ones even after they have been removed from operation, e.g., by a system administrator who learns of the misbehavior. We would like to limit such interference so that, after only a limited number of writes by good clients, any lurking writes left behind by a bad client will no longer be visible to good clients. A lurking write is a modification launched by the bad client before it was removed from operation that will become visible (possibly with help from an accomplice) after it has left the system. By limiting such writes we can ensure that the object becomes useful again after the departure of the bad client, e.g., some invariant that good clients preserve will hold.

Of course, it is not possible to prevent actions by a bad client, even if it has been shut down, if the private key it uses to prove that it is authorized to modify an object can be used by other nodes; thus, we consider a bad client to be in the system as long as any node knows its private key. (In practice this problem might be handled by an administrator 
removing the bad client from the access control list.)

Thus, we make the following contributions:

- We present strong correctness conditions for an atomic $\mathrm{read} /$ write object in a system in which both clients and replicas can be Byzantine. Our conditions improve on existing correctness conditions $[6,11]$ by ensuring atomicity for good clients, and also imposing strict limits on the effects of bad clients that have been removed from operation: our conditions bound the number of lurking writes by a constant factor, and prevent lurking writes after bad clients stop and good clients subsequently overwrite the data.

- We present the first Byzantine quorum protocols that satisfy the conditions using only $3 f+1$ replicas (to survive $f$ faulty replicas) and work in an unreliable asynchronous network like the Internet. Furthermore our protocols are efficient: To do writes requires either 3 phases (our base protocol) or mostly 2 phases (our optimized protocol). Reads usually require 1 phase; they sometimes need an additional phase (to write back the data just read). Our base protocol ensures that there can be at most one lurking write after a bad client has left the system; the optimized protocol ensures slightly weaker behavior: there can be a most two lurking writes.

- We are able to manage with a small number of replicas and phases because our protocol makes use of "certificates", an approach that we believe can be used to advantage in other protocols. A certificate is a collection of $2 f+1$ authenticated messages from different replicas that vouch for some fact, e.g., that a client has completed its previous write, or that the state read from a single replica is valid.

- We prove the correctness of our protocols, both safety and liveness. In addition we describe a variation of our protocols that supports a still stronger correctness condition, in which we can bound the number of writes of good clients that it takes to ensure that modifications of the bad client that has left the system will no longer be visible. This variation sometimes requires an additional phase to do a write.

The rest of this paper is organized as follows. We begin by describing our assumptions about the system. Section 3 describes our base protocol. Section 4 describes our correctness conditions and we prove our base protocol meets them in Section 5. Section 6 describes our optimized protocol and proves its correctness. Section 7 describes a variation of our protocols that allows us to bound the number of overwrites needed to hide effects of lurking writes. Section 8 discusses related work and we conclude in Section 9.

\section{Model}

The system consists of a set $\mathcal{C}=\left\{c_{1}, \ldots, c_{n}\right\}$ of client processes and a set $\mathcal{S}=\left\{s_{1}, \ldots, s_{n}\right\}$ of server processes. Client and server processes are classified as either correct or faulty. Correct processes are constrained to obey their specification, i.e., they follow the prescribed algorithms. Faulty processes may deviate arbitrarily from their specification, i.e., we assume a Byzantine failure model [8]. Note that faulty processes include those that fail benignly as well as those suffering from Byzantine failures.

We refer to the set of faulty clients as $\mathcal{C}_{\text {bad }}$ and the set of correct clients as $\mathcal{C}_{o k}$, and consequently we have $\mathcal{C}=$ $\mathcal{C}_{\text {bad }} \cup \mathcal{C}_{o k}$ (and respectively $\mathcal{S}=\mathcal{S}_{\text {bad }} \cup \mathcal{S}_{o k}$ ). Note that our algorithms do not require the knowledge of $\mathcal{C}_{o k}, \mathcal{C}_{\text {bad }}$, $\mathcal{S}_{\text {ok }}$, nor $\mathcal{S}_{\text {bad }}$. In other words, we do not assume that we can detect faults.

We assume an asynchronous distributed system where nodes are connected by a network that may fail to deliver messages, delay them, duplicate them, corrupt them, or deliver them out of order, and there are no known bounds on message delays or on the time to execute operations. We assume the network is fully connected, i.e., given a node identifier, any other node can (attempt to) contact the first node directly by sending it a message.

For liveness, we only require that if a client keeps retransmitting a request to a correct server, the reply to that request will eventually be received.

We assume nodes can use unforgeable digital signatures to authenticate communication. More precisely, any node, $n$, can authenticate messages it sends by signing them. We denote a message $m$ signed by $n$ as $\langle m\rangle_{\sigma_{n}}$. And no node can send $\langle m\rangle_{\sigma_{n}}$ (either directly or as part of another message) on the network for any value of $m$, unless it is repeating a message that has been sent before or it knows $n^{\prime} s$ private key. (In some cases we will be able to replace costly digital signatures with point to point authenticators, as discussed later, but we will maintain the notation $\langle m\rangle_{\sigma_{n}}$.)

We assume the existence of a collision-resistant hash function, $h$, such that any node can compute a digest $h(m)$ of a message $m$ and it is impossible to find two distinct messages $m$ and $m^{\prime}$ such that $h(m)=h\left(m^{\prime}\right)$.

To avoid replay attacks we tag certain messages with nonces that are signed in the replies. We assume that when clients pick nonces they will not choose a repeated nonce.

\section{BFT-BC Algorithm}

This section presents our construction for a read/write variable implemented using Byzantine quorum replication, and that tolerates Byzantine-faulty clients. We begin by giving a brief overview of Byzantine quorums in Section 3.1. Then we present our base protocol. This protocol requires 
3 phases to write; we discuss its costs in Section 3.3. Section 6 presents an optimization that requires only 2 phases most of the time.

\subsection{Byzantine Quorums Overview}

This section gives an overview of how current algorithms use Byzantine quorums to implement a shared read/write variable. The presentation follows the original $\mathrm{BQS}$ protocol [9], using their construction for a system that doesn't handle Byzantine clients. (We discuss this system further in Section 8.)

A Byzantine quorum system defines a set of subsets of a replica group with certain intersection properties. A typical way to configure such a system is to use groups of $3 f+1$ replicas to survive $f$ failures with quorums of size $2 f+1$ replicas. This ensures that any two quorums intersect in at least one non-faulty replica. Each of the replicas maintains a copy of the data object, along with an associated timestamp, and a client signature that authenticates the data and timestamp.

Two phases are required to write the data. First, the client contacts a quorum to obtain the highest timestamp produced so far. The client then picks a timestamp higher than what was returned in the first phase, signs the new value and timestamp, and proceeds to the second phase where the new value is stored at a quorum of replicas.

Replicas allow write requests only from authorized clients. A replica overwrites what it has stored only if the timestamp in the request is greater than what it already has.

The read protocol usually has a single phase where the client queries a quorum of replicas and returns the value with the highest timestamp (provided the signature is correct). An extension of this protocol [10] requires a second phase that writes back the highest value read to a quorum of replicas (this ensures atomic semantics).

\subsection{BFT-BC Protocol}

The protocol just presented is not designed to handle Byzantine-faulty clients, which can cause damage to the system in several ways, e.g.:

1. Not follow the protocol by writing different values associated with the same timestamp.

2. Only carry out the protocol partially, e.g., install a modification at just one replica.

3. Choose a very large timestamp and exhaust the timestamp space.

4. Issue a large number of write requests and hand them off to a colluder who will run them after the bad client has been removed from the system. This colluder could be one of the replicas, or a completely separate machine.
These actions can cause unwanted behavior from Byzantine quorum protocols. In particular, we want to achieve a protocol where faulty clients cannot prevent good clients from making progress, but also where, once the bad client stops, the number of lurking writes seen by that bad client is bounded by a small constant. (We define these conditions formally in Section 4.)

Our protocol prevents Byzantine clients from the above mentioned actions, thereby accomplishing these correctness conditions. It uses $3 f+1$ replicas, and quorums can be any subset with $2 f+1$ replicas. It uses a three-phase protocol to write, consisting of a read phase to obtain the most recent timestamp, a prepare phase in which a client announces its intention to write a particular timestamp and value, and a write phase in which the client does the write that it prepared previously.

As it moves from one phase to another, however, the client needs to "prove" that what it is doing is legitimate. It does this by using certificates. A certificate takes the form of a quorum of authenticated messages from different replicas that vouch for some fact.

For example, the purpose of the prepare phase is for the client to inform the replicas of what it intends to do, and this must be acceptable, e.g., the timestamp it proposes cannot be too big. Replicas that approve the prepare request return messages that together provide a prepare certificate. This certificate is needed to carry out the write phase: a client can only carry out a write that has been approved. When a write is performed by a replica it returns an authenticated message and these messages together form a write certificate. This certificate is needed for the replica to do its next write: it cannot complete the prepare phase for a second write (with a higher timestamp) without completing its first one. This constraint, plus the fact that certificates cannot be forged or predicted in advance by bad clients, is what limits the number of lurking writes a bad client can leave behind when it stops (to be carried out by some node that colludes with it).

We now describe our protocol in detail.

As mentioned, each object in BFT-BC is replicated at a set of $3 f+1$ replicas, numbered from 0 to $3 f$. Quorums can be any subset with $2 f+1$ replicas and we use a three-phase protocol to write.

A valid prepare certificate contains a quorum of statements $\langle\text { PREPARE-REPLY, } t s, h\rangle_{\sigma_{r}}$ where each statement is authenticated by its replica $r$ and all statements contain the same timestamp $t s$ and hash $h$. A valid write certificate contains a quorum of statements $\langle\text { WRITE-REPLY, } t s\rangle_{\sigma_{r}}$ where each component is authenticated by its replica $r$ and all statements contain the same timestamp $t s$. Given a certificate $c$, we use the notation $c . t s$ to denote the timestamp in that certificate, and we use the notation $c . h$ to denote the hash in a prepare certificate $c$. 
Protocol at client $c$ to write value val.

- phase 1 .

1. send $\langle$ READ-TS, nonce $\rangle$ to all replicas

2. wait for a quorum of valid (well-formed, and correctly authenticated) replies of the form $\angle$ READ-TSREPLY, $p$, nonce $\rangle_{\sigma_{r}}$, authenticated by the replier, where $p$ is a correct prepare certificate (well-formed, and all signatures verify).

Select $P_{\max }$, the certificate containing the largest timestamp.

- phase 2

1. send $\left\langle\text { PREPARE, } P_{\text {max }}, t, h(\mathrm{val}), W_{\text {cert }}\right\rangle_{\sigma_{c}}$, authenticated by $c$, to all replicas. Here $t=\operatorname{succ}\left(P_{\max } . t s, c\right)$, and Wcert is the write certificate of $c$ 's last write (as explained later) or null if this is $c$ 's first write.

2. wait for a quorum of valid (well-formed, correctly signed, with matching values for $h$ and $t$ ) replies of the form $\langle\text { PREPARE-REPLY, } t, h\rangle_{\sigma_{r}}$. These replies form a prepare certificate $P_{\text {new }}$ for $h$ and $t$.

- phase 3 .

1. send $\left\langle\text { WRITE, val, } P_{n e w}\right\rangle_{\sigma_{c}}$ to all replicas.

2. wait for a quorum of valid (well-formed, and correctly signed) replies of the form 〈WRITE-REPLY, $t\rangle_{\sigma_{r}}$. These replies form a write certificate, which the client retains for its next write.

\section{Figure 1. Client write protocol (pseudocode).}

Each replica keeps the following per-object information:

- data, the value of the object.

- $P_{c e r t}$, a valid prepare certificate for $h($ data $)$.

- $P_{\text {list }}$, a set of tuples $\langle t, h, c\rangle$ containing the timestamp, hash, and client identifier of proposed writes.

- write $_{T S}$, the timestamp of the latest write known to have completed at $2 f+1$ replicas.

Our system can deal with multiple objects; each object would have a distinct identifier and each read and write would identify the object of interest. To simplify the presentation, however, we consider a system containing only a single object, and therefore we omit object identifiers from the description of the protocol.

\subsubsection{Write Protocol}

Our protocols require that different clients choose different timestamps, and therefore we construct timestamps by concatenating a sequence number with a client identifier: $t s=\langle t s . v a l, t s . i d\rangle$. We assume that client identifiers are unique. To increment a timestamp a client with identifier $c$ uses the following function: $\operatorname{succ}(t s, c)=\langle t$ s.val $+1, c\rangle$.

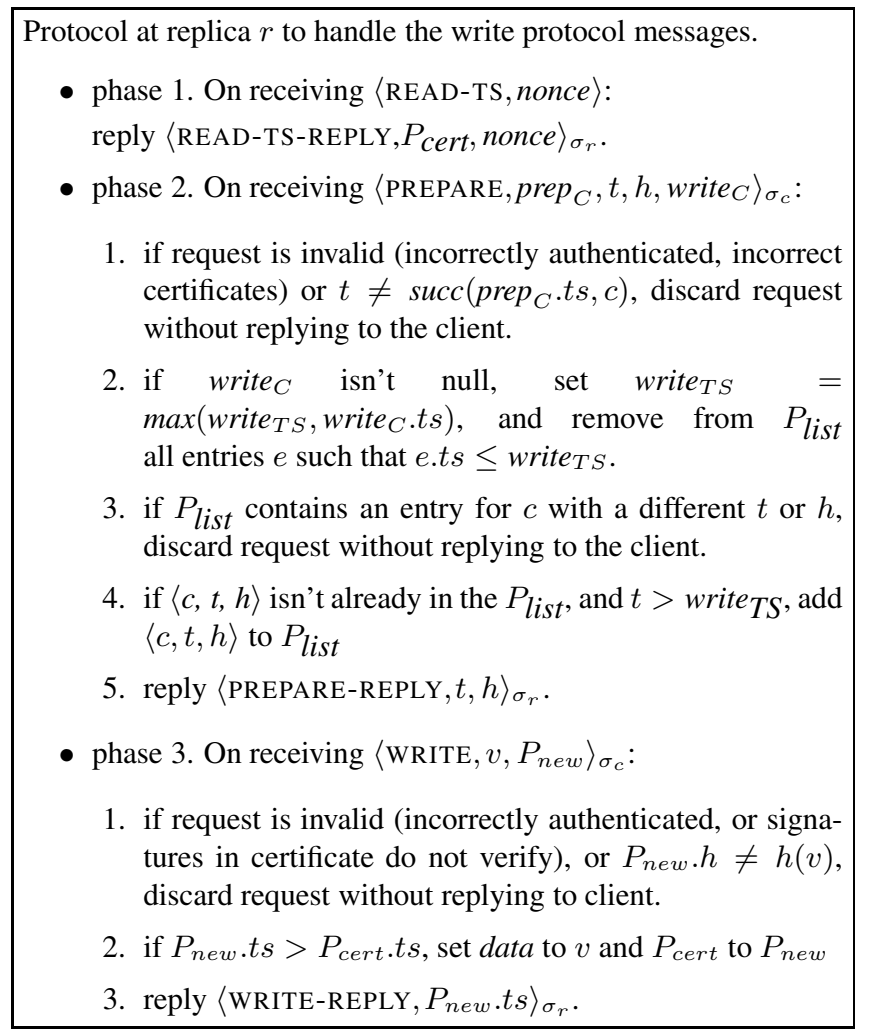

Figure 2. Replica write protocol.

Timestamps can be compared in the usual way, by comparing the val parts and if these agree, comparing the client ids.

Figures 1 and 2 give the pseudocode of our three-phase write protocol, for the client and replicas respectively. In all phases, clients retransmit their requests to account for lost messages; they stop retransmitting once they collect a quorum of valid replies. (Note that we use only client retransmission; this handles the loss of both client request messages and replica replies.)

Phase 2 processing at the replica is the crucial part of the algorithm. The replicas check to ensure that the timestamp being proposed is correct, that the client is doing just one prepare, that the value being proposed does not differ from a previous request for the same timestamp, and that the client has completed its previous write.

\subsubsection{Read Protocol}

The read protocol usually requires just one phase.

Phase 1. The client sends a $\langle\mathrm{READ}$, nonce $\rangle$ request to all replicas. A replica replies with its value, prepare certificate, and nonce, all authenticated by it. The client waits for a quorum of valid responses and chooses the one with the largest timestamp (this is the return value). If all the timestamps are the same the read protocol ends. 
Phase 2. Otherwise the client performs the write-back phase for the largest timestamp; this is identical to phase 3 of writing, except that the client needs to send only to replicas that are behind, and it must wait only for enough responses to ensure that $2 f+1$ replicas now have the new information.

The read protocol uses client retransmission to account for lost messages, as in the write operations.

\subsection{Protocol Costs}

\subsubsection{State and Message Complexity}

The amount of state stored by the BFT-BC algorithm is small: the only state components that are non-constant are the prepare list, which is a set of timestamps and hashes of prepared writes, and the prepare certificate stored at each replica.

The size of the prepare list is $O(|\mathcal{C}|)$, where $|\mathcal{C}|$ is the number of allowed writers. Generally this will not be a large number, but in addition the list is small because when replicas receive write certificates in phase 2 , they remove old entries with a lower timestamp than the one in the write certificate. We could speed up removing entries from the list if we propagated write certificates in more messages, e.g., in read requests.

The size of the prepare certificate is $O(|Q|)$, where $|Q|=$ $2 f+1$.

The number of messages exchanged by an operation in BFT-BC is $O(|Q|)$, since each operation consists of three RPCs (i.e., a sequence of a request message and the respective reply) to a quorum of replicas, assuming no retransmissions are required. The total message size for each operation is $O\left(|Q|^{2}\right)$, because some of the messages contain certificates whose size is $O(|Q|)$.

\subsubsection{Cost of Authentication}

In the above description we mentioned that certain messages or statements were authenticated, but the kind of authentication that may be used was unspecified. This issue is important since different techniques have different costs: we can authenticate a point-to-point message by means of symmetric cryptography by establishing session keys and using message authentication codes (MACs). This does not work for signing statements that have to be shown to other parties, in which case we need to rely on more expensive public key cryptography.

Our protocol requires signing using public key cryptography in two places: the phase 2 and phase 3 responses. These signatures are needed because they are used as proofs offered to third parties, e.g., the prepare certificate is generated for one client but then used by a different client to justify its choice of the next timestamp.
A further point is that only the phase 2 response signature needs to happen in the foreground. The signature for the phase 3 response can be done in the background: a replica can do this after replying to the phase 2 request, so that it will have the signature ready when the phase 3 request arrives.

\section{Correctness Condition}

This section defines the correctness conditions for a variable shared by multiple clients that may incur Byzantine failures. We begin by defining histories (Section 4.1), and then we give our correctness condition.

\subsection{Histories and Stopping}

We use a definition of history similar to the one proposed in [6], extended to handle Byzantine clients.

The execution is modeled by a history, which is a sequence of events of the following types:

- Invocation of operations.

- Response to operation invocations.

- Stop events.

An invocation by a client $c$ is written $\langle c: x . o p\rangle$ where $x$ is an object name and $o p$ is an operation name (possibly including arguments). A response to $c$ is written $\langle c: x$.rtval $\rangle$ where rtval is the response value. A response matches an invocation if their object names agree and their client names agree. A stop event by client $c$ is written $\langle c: s t o p\rangle$.

A history is sequential if it begins with an invocation, if every response is immediately followed by an invocation (or a stop or no event), and if every invocation is immediately followed by a matching response. A client subhistory $H \mid c$ of a history $H$ is the subsequence of all events in $H$ whose client names are $c$. A history is well-formed if for each client $c, H \mid c$ is sequential. We use $\mathcal{H}$ to denote the set of wellformed histories.

An object subhistory $H \mid x$ of a history $H$ is the subsequence of all events whose object names are $x$ and a history $H$ is a single-object history for some object $x$ if $H \mid x=H$. A sequential specification for an object is a prefix-closed set of single-object sequential histories for that object. A sequential history $H$ is legal if each object subhistory $H \mid x$ belongs to the sequential specification of $x$.

An operation $o$ in a history is a pair consisting of an invocation $\operatorname{inv}(o)$ and the next matching response $r s p(o)$. A history $H$ induces an irreflexive partial order $<_{H}$ on the operations and stop events in $H$ as follows: $o_{0}<_{H} o_{1}$ if and only if $r s p\left(o_{0}\right)$ precedes $i n v\left(o_{1}\right)$ in $H ; o_{0}<_{H}\langle c: s t o p\rangle$ if and only if $r s p\left(o_{0}\right)$ precedes $\langle c: s t o p\rangle$ in $H ;\langle c: s t o p\rangle<_{H}$ $o_{1}$ if and only if $\langle c: s t o p\rangle$ precedes $i n v\left(o_{1}\right)$ in $H$; and $\left\langle c_{1}:\right.$ stop $\rangle<_{H}\left\langle c_{2}:\right.$ stop $\rangle$ if and only if $\left\langle c_{1}:\right.$ stop $\rangle$ precedes $\left\langle c_{2}:\right.$ stop $\rangle$ in $\mathrm{H}$. 


\subsubsection{Verifiable Histories}

The notion of linearizability [6] is applied to a concurrent computation, which is modeled by a history (a finite sequence of invocation and response events by all processes).

It would be difficult to model such computations in our environment, since faulty processes do not obey any specification, and therefore we cannot define what an invocation or a response means for such processes. However, we know that after a STOP event from a faulty process it will halt, meaning that it can no longer produce correctly signed messages (although we can still observe the replay of old messages after a STOP event). In practice this may correspond to different scenarios, e.g., an administrator removing the node's public key from the system's access control list (this occurrence implies a stronger notion of STOP where replays are also discarded, but our correctness condition and the correctness of our algorithms do not require this); or turning off the node before it can leak its private key, even if the node leaked several signed messages before halting (e.g., this might be enforced using a secure coprocessor [7], but we do not require their use).

Therefore we introduce the concept of a verifiable history which is a history that contains the sequence of invocations and responses from correct clients, and stop events from faulty clients.

The correctness condition we present next is applicable only to verifiable histories.

\subsection{Correctness Condition}

We are now ready to define the correctness condition for variables shared by multiple processes that may incur Byzantine failures.

The idea is that, as in linearizability [6], we require that the verifiable history looks plausible to the correct processes, i.e., that there is a sequential history in which all processes are correct that explains what the correct processes observed. Furthermore, we require that once a faulty client stops, its subsequent effects on the system are limited in the following way: The number of operations by that client that are "seen" by correct clients after it stopped is bounded by some constant.

These concepts can be formalized as follows.

Definition 1 A verifiable history $H \in \mathcal{H}$ is BFT-linearizable if there exists some legal sequential history $H^{\prime} \in \mathcal{H}$ such that

$$
\begin{aligned}
& \text { 1. } H\left|p=H^{\prime}\right| p, \forall p \in \mathcal{C}_{o k} \\
& \text { 2. }<_{H} \subseteq<_{H^{\prime}} \\
& \text { 3. } \forall c \in \mathcal{C}_{\text {bad }}:\langle c: \text { stop }\rangle \in H \Rightarrow \\
& \left(\exists h_{1}, h_{2} \subseteq H^{\prime}: H^{\prime}=h_{1}\langle c: \text { stop }\rangle h_{2} \wedge\right. \\
& \left.\left|\left\{o \in h_{2}: o=\langle c: x . o p\rangle\right\}\right| \leq \text { max }-b\right)
\end{aligned}
$$

Points 1 and 2 of the definition above state that there must exist a sequential history that looks the same to correct processes as the verifiable history in question, and that sequential history must preserve the $<_{H}$ ordering (in other words, if an operation or a stop event precedes another operation or stop event the verifiable history, then the precedence must also hold in the sequential history).

Point 3 says that if a faulty client $c$ stops, then when we look at the sequential history and consider the sub-history after the stop event by $c$ (this is $h_{2}$ ), the number of events by client $c$ in that history is bounded by $\max -b$.

Note that there is a counter-intuitive notion that a bad client can execute operations after it has stopped. This corresponds to the notion that the bad client left some pending operations (e.g., with a colluder) before it left, and this is precisely the effect we want to minimize.

We can now define a BFT-linearizable object to be an object whose verifiable histories are BFT-linearizable with respect to its sequential specification.

\section{Correctness Proof}

This section sketches a proof that the algorithm presented in Section 3 meets the correctness conditions presented in Section 4.

The idea is to look at what kind of properties we can ensure given a certain state of the system when a faulty client $c$ stops. If we look at the current-ts values stored at that instant, we can guarantee the following.

Lemma 1. Let $t s_{\max }$ be the $f+1$ st highest timestamp stored by non-faulty replicas at time $t_{\text {stop }}$ (when some faulty client $c$ stops). Then the following must hold

1. At any time up to $t_{\text {stop }}$, no node can collect a write certificate for a timestamp $t^{\prime}$ such that $t^{\prime}>t s_{\max }$.

2. There are no two timestamps $t_{1}, t_{2}>t s_{\max }$ such that client $c$ assembled a prepare certificate for $t_{1}$ and $t_{2}$.

3. No two prepare certificates exist for the same timestamp $t>t s_{\max }$ and different associated values.

\section{Proof.}

1. By algorithm construction, a nonfaulty replica will not sign an entry in a write certificate vouching for a timestamp higher than the one held in the variable current-ts. Since non-faulty replicas always store increasing timestamp values, this means that the number of signatures that can be held in the system at time $t_{\text {stop }}$ for timestamps higher than tsmax is at most $2 f$ (i.e., $f$ from faulty replicas and the $f$ correct replicas that may hold timestamps higher than $t s_{\max }$ ).

2. By contradiction, suppose that client $c$ held prepare certificates for $t_{1}, t_{2}$, both greater than $t_{\text {max }}$. The two certificates intersect in at least one nonfaulty replica. By 
part (1) of this lemma, that replica had its write-ts variable always at a value less than or equal to $t s_{\max }$ (at all times up to and including $t_{\text {stop }}$ ). Therefore that replica could not have signed its entry in both certificates, since after signing the first one (say, for $t_{1}$ ) it would insert an entry for client $c$ and $t_{1}$ in its prepare list, and that entry would not be removed (because of the value of write-ts), which prevents the replica from signing its entry in the certificate for $t_{2}$.

3. Suppose, by contradiction that two prepare certificates exist for timestamp $t$ and values $v$ and $v^{\prime}$. By the quorum intersection properties, these two prepare certificates contain at least one signature from the same correct replica. By part (1) of this lemma, no write certificate was ever assembled for timestamps greater or equal than $t s_{\max }$, and these entries in the prepare list were never removed. This violates the constraint that correct replicas do not sign a timestamp that is already in its prepare list for a different value. rithm.

We are now ready to show the correctness of our algo-

Theorem 1. The BFT-BC algorithm is BFT-linearizable.

Proof. Consider any correct reader in the case that the writer is correct. In this case, the quorum initially accessed in a read operation intersects the quorum written in the most recently completed write operation in at least one correct replica. Therefore, the read returns either the value in the most recently completed write, or a value with a higher timestamp (which could be written concurrently with the read). Since a read also writes back its obtained value and timestamp to a quorum of processes, any subsequent read by a correct reader will return that timestamp value or a later one. So, for any execution, we construct the history needed to show BFT-linearizability by putting every read right after the write whose value it returns.

If the writer is faulty, we construct the sequential history to show BFT-linearizability as follows: for each read by a correct reader returning $v$ such that the phase 3 request for $v$ was produced by client $c_{b}$, insert a write operation in the history that writes $v$ (by client $c_{b}$ ) immediately before the read.

Insert a stop event before the invocation of the first operation that succeeded the stop event in the original (verifiable) history (i.e., as late as possible while preserving the $<_{H}$ dependencies).

It is left to show that if a faulty process stops, this history contains a bounded number of operations by that faulty process after it stops.

To prove this condition we note that Lemma 1 part (2) says that client $c$ only assembled prepare certificates for a single timestamp for a write that would become visible after it stopped (i.e., with a timestamp greater than $t s_{\max }$ ), and Lemma 1 part (3) implies that if the write were to become visible, the prepare certificate could only correspond to a single value. This means the number of operations by the faulty client $c$ after it stops is at most one.

\subsection{Liveness}

As far as liveness is concerned, we guarantee that good clients can always execute read operations in the time it takes for two client RPCs to complete at $2 f+1$ replicas (i.e., the requests reaching the replicas and the respective replies returning to the client). This is so because at least $2 f+1$ replicas will provide them with the appropriate answers (client requests are answered unconditionally provided the requests are well-formed). For the write protocol, the operation will complete in the time for three client RPCs to complete in $2 f+1$ replicas, and the trick is to ensure that their phase 2 requests are never refused (since phase 1 requests are answered unconditionally and phase 3 requests are answered if they contain a valid prepare certificate, which good clients will always send).

The phase 2 request also gets replies since the client will submit a correct timestamp and hash, plus the write certificate for its latest write; the latter allows the replica to discard the client's entry in the prepare list and accept the prepare request.

\section{Optimized BFT-BC Algorithm}

This section describes our optimized protocol, which usually requires only two phases to do a write, and proves that the optimized protocol satisfies our correctness condition.

\subsection{Optimized BFT-BC}

Our plan is to avoid one of the phases by merging it with another phase. We cannot merge phases 2 and 3 since the protocol requires that the prepare information be stored at $f+1$ honest replicas before the client can do phase 3 : this is how we avoid a bad client being able to create many writes in advance that could be launched after it leaves the system. Therefore, we will merge phases 1 and 2 .

The idea is that the client sends the hash in the phase 1 request and the replica does phase 2 on its behalf: it predicts the next timestamp, adds the new timestamp with the hash to the prepare list, and returns this information, signed by itself. If the client receives a quorum of responses all for a particular new timestamp, it can move to phase 3 immediately.

This optimization will work well in the normal case where writes are received by all replicas in the same order. Therefore the good client is highly likely to receive a 
quorum of replies for the same timestamp in the responses in phase 1 , and most of the time a write will require two phases.

However there are problems that must be overcome for the optimization to be used. They occur when there are concurrent writes or when other clients are faulty.

A simple example is the following. Suppose a client does phase 1 for some hash $h$. It receives a PREPARE-REPLY for a particular timestamp $t$ from replicas $R 1$ and $R 2$, and a PREPARE-REPLY for a larger timestamp $t^{\prime}$ from replicas $R 3$ and $R 4$. The client will then be unable to get approval for either $t$ or $t^{\prime}$, because to approve the request a replica would need to violate our restriction on having at most one entry per client on the prepare list.

Clearly we need to find a way to allow clients to do writes in cases like this one. But given our current constraints this is not possible.

To allow the client to make progress we will weaken our constraint on how many entries a client can have in the prepare list. In particular we will change the replica state to have a second list of prepared writes, opt ${ }_{\text {list }}$, with the constraint that a client can have at most one entry per list. The two entries for the same client (one in each list) might be for different timestamps, or they might be for the same timestamp. In the latter case, it will be possible for the write with that timestamp to happen twice (when there is a bad client). In this case we need a way to order the writes: we will do this by using the numeric order on their hashes.

\subsection{Detailed Write Protocol}

Phase 1. The client, $c$, sends a READ-TS-PREP request containing the hash of the proposed value, and the client's current write certificate, to all replicas.

Each replica processes the request in the usual phase2 way, including removing entries from the prepare lists. Then it will do the prepare on behalf of the client for timestamp $t^{\prime}=\operatorname{succ}(t s, c)$, unless the client already has an entry in either prepare list for a different timestamp or hash. If the prepare is successful, the replica adds an entry to the opt $t_{\text {list }}$ for $t^{\prime}$ and the hash (unless that entry is already in the list) and returns a PREPARE-REPLY signed by it; otherwise it returns a normal phase 1 response.

If the client gets a quorum of PREPARE-REPLY (i.e., obtains a prepare certificate) for the same new timestamp, it moves to phase 3.

Phase 2. Otherwise the client chooses the new timestamp as before and carries out phase 2 for it (as in the normal protocol). When it has a quorum of signatures for this choice (obtained either in phase 1 or phase 2), it moves to phase 3 .

Replicas handle phase 2 requests as in the normal protocol; they ignore the opt list in this processing.

Phase 3. The client does phase 3 in the normal way.
The replica also does normal processing, except that the timestamp in the write might match the current timestamp, but with a different value: in this case it retains the value with the larger hash.

\subsection{Discussion}

The optimized protocol can lead to a client having entries on two prepare lists (normal and optimized). A dishonest client can exploit this to carry out phase 3 twice as part of the same write request. And as a result, each dishonest client can leave two lurking writes behind when it is removed from the system.

Another point is that it is now possible for honest clients to see valid responses to a read request that have the same timestamp but different values. The client protocol resolves this situation by returning (and writing back) the value with the larger hash.

\subsection{Optimized BFT-BC Correctness}

For the optimized protocol some of the invariants shown in Section 5 need to be slightly modified. Notably, Lemma 1 parts (2) and (3) no longer hold, since a faulty client can now collect two distinct prepare certificates (for its entries in the prepare list and opt $_{\text {list }}$ ). Therefore Lemma 1 parts (2) and (3) become:

Lemma 1' (2). For the optimized BFT-BC protocol, no more than two prepare certificates can exist for distinct timestamp, value pairs, with timestamps greater than $t s_{\max }$. Proof. Same as Lemma 1, but taking into consideration that the new algorithm allows for one entry in the normal prepare list, and another in the optimistic prepare list.

This only affects the proof of Theorem 1 in that the number of operations by a faulty client after it stops becomes 2 instead of 1, and therefore the main theorem still holds:

Theorem 2. The optimized BFT-BC algorithm is BFTlinearizable.

\section{Stronger Correctness Conditions}

In this section we propose a stronger correctness condition than the one introduced in Section 4, and discuss how to extend the current protocol to meet the new condition.

\subsection{New Correctness Conditions}

The idea is that we want to further limit the effects a bad client can have on the system once it stopped such that, if correct clients execute a constant number of write operations after a faulty client stops, then no more operations by that client will ever be "seen". (We can generalize this to 
any state-overwriting operation in a set $O_{\text {overwrite }}$ in case the variable has an interface other than read and write.)

We formalize the BFT-linearizable+ condition as being equal to the condition presented in Section 4, except that point (3) is modified to state that no operations by the faulty client $c$ appear after the $k$ th consecutive state-overwriting operation in $h_{2}$ (where $k=O(1)$ ).

\subsection{Modified BFT-BC protocol}

The BFT-BC protocols do not meet this stronger correctness condition because a set $C$ of colluding clients can prepare a series of $|C|$ writes with successive timestamps, leaving a lurking write that requires $|C|$ writes by correct clients to ensure that the lurking write will no longer be seen.

To address this issue, we need to modify the BFT-BC protocol to require the client to submit a write certificate in its prepare request (along with the information it already sends in the prepare). This certificate ensures that the timestamp it is proposing is the successor of one that corresponds to a write that has already happened; a replica will discard the request if this condition does not hold.

The client can easily assemble this certificate in phase 1 if all responses to its phase 1 request contain the same timestamp. If they don't, the client can obtain the certificate by doing a write-back; the difficulty is getting the value to write back. This could be accomplished by having phase 1 actually be a read, but this is unattractive since values can be large. So instead, the client fetches the value, if necessary, in a separate step; effectively it redoes phase 1 as a normal read, although this can be optimized (e.g., to fetch from the $f+1$ replicas that returned the largest timestamps).

This scheme guarantees that the timestamp in the lurking write is the successor of a lower bound on the value stored by at least $f+1$ non-faulty replicas when the bad client stopped. Consequently, if there were two successive writes by correct clients after the bad client stopped, the lurking write would no longer be seen.

\section{Related Work}

In this section we discuss the previous work that dealt with Byzantine clients, and also the previous work on correctness conditions.

Our protocol is more efficient than those proposed previously. Furthermore it enforces stronger constraints on the behavior of Byzantine clients: we handle all problems handled by previous protocols plus we limit the number of lurking writes that a Byzantine client can leave behind after it has left the system.

In addition we provide stronger liveness guarantees than previous protocols: in particular read operations cannot return null values, and reads terminate in a constant number of rounds, independently of the behavior of concurrent writers.

The initial work on Byzantine quorum systems [9] described the quorum protocol discussed in Section 3, which used $3 f+1$ replicas, one phase reads and two phase writes, and did not handle Byzantine clients. That paper also described a protocol for a system of $4 f+1$ replicas that prevented malicious clients from associating different values with different timestamps; the protocol required a threephase communication among the replicas to be carried out as part of performing a write (in addition to the client-server communication) where servers guarantee that each value and timestamp pair is propagated to a quorum. The protocol provides liveness, but at the expense of providing weak semantics for reads where they could return a null value in case of concurrent writes.

The Phalanx system [10] improves on the previous result in two ways. First it added the write-back phase (to the simpler protocol) as a way to ensure atomicity for reads. In addition it presented a more efficient protocol to handle Byzantine clients. That protocol used $4 f+1$ replicas, but clients carried out a three-phase protocol to do a write, and the server-to-server communication was no longer needed. In the new protocol for Byzantine clients, read operations could return a null value if there was an incomplete or a concurrent write.

The work by Goodson et al. [5] proposes a solution for erasure-coded storage that tolerates Byzantine failures of clients and servers; this system requires $4 f+1$ replicas. This work is focused on integrating erasure coded storage with Byzantine quorum protocols and they tolerate Byzantine clients that write different "values" to different replicas by having the next reader detect the inconsistency and, if possible, repair it. In some cases, reads may return null.

The work of Martin et al. [12] proposes a protocol that only uses $3 f+1$ replicas (like our protocol). They require a quorum of $2 f+1$ identical replies for read operations to succeed, which is difficult to ensure in an asynchronous system. Their solution is to assume a reliable asynchronous network model, where each message is delivered to all correct replicas. This means that infinite retransmission buffers are needed in an asynchronous environment like the Internet: the failure of a single replica (which might just have crashed) causes all messages from that point on to be remembered and retransmitted. In this protocol concurrent writers can slow down readers. The authors discuss how to extend their protocols to guarantee that Byzantine clients associate the same value with the same timestamp by having servers forward the writes among themselves (again, this is possible due to their network model) and keep the highest value for each timestamp. They also discuss how to prevent a faulty client from exhausting resources at correct servers, but at the expense of possibly sacrificing liveness for correct readers. 
Two papers (Bazzi and Ding [2], Cachin and Tessaro [3]) describe protocols that enforce non-skipping timestamps. In both cases, the protocols are based on the Martin et al. work, which uses the reliable asynchronous network model with the problems we mentioned above. Furthermore these papers do not try to address the issue of lurking writes. The protocol of Bazzi and Ding [2] requires $4 f+1$ replicas, while Cachin and Tessaro [3] require $3 f+1$ replicas.

Attiya and Bar-Or [1] present quorum constructions that tolerate a weaker form of Byzantine failures: semiByzantine clients. These clients can fail either by stopping, or by writing an erroneous value to a shared register some number of times, but otherwise follow the protocol. We improve on this paper by considering a more generic fault model for clients.

Our definition of BFT-linearizability presented in Section 4 builds upon a condition (called Byznearizability) proposed by Malkhi et al. [11]. Their work was the first to point out the problem of lurking writes. However, their correctness condition is weaker than ours, since they require only that the number of lurking writes is finite, whereas we require that the number of lurking writes is bounded by a constant (one or two in the case of our protocols). In fact, their correctness condition is met by the variant of the Phalanx protocol [10] that was designed to handle only honest clients. Another point is that Byznearizability did not consider the possibility that malicious clients might halt before they could leak their private keys, and therefore Byznearizability requires all faulty clients and servers to stop in order to provide any guarantees. Our condition is also stronger due to the fact that we consider the number of overwrites to mask all the lurking writes.

\section{Conclusions}

This paper has presented novel protocols for Byzantine quorum systems that provide atomic semantics despite Byzantine faulty clients. Our protocols are more efficient and handle more problems caused by Byzantine clients than previous proposals. We prevent bad clients from leaving behind more than one or two lurking writes and from exhausting the timestamp space. In addition Byzantine clients are unable to interfere with good clients in the sense that they cannot prevent good clients from completing reads and writes, and they cannot cause good clients to see inconsistencies. Another point is that an extension of our protocol can additionally ensure that the effects of a Byzantine client are no longer visible to good clients at all after two successive writes by good clients (or four successive writes in the optimized protocol).

We also presented strong correctness conditions that address the above problems: we require that protocols guarantee atomicity for good clients, and limit what can be done on behalf of bad clients once they leave the system. Furthermore we proved that our protocols are both safe (they meet those conditions) and live.

Our protocols are designed to work in an unreliable asynchronous system like the Internet and they are highly efficient. Our base protocol completes writes in three network round-trips; the optimized protocol reduces this cost so that writes normally complete in two network round-trips (at the expense of allowing one more lurking write). In either case reads normally complete in one phase, and require no more than two phases, no matter what the bad clients are doing. We achieve these efficiencies because of our use of certificates, which allow clients or replicas to know that information presented to them is valid, without having to hear the same thing directly from $2 f+1$ replicas.

\section{Acknowledgments}

We would like to thank Rida Bazzi, Christian Cachin, and the anonymous reviewers for helpful feedback.

\section{References}

[1] H. Attiya and A. Bar-Or. Sharing memory with semibyzantine clients and faulty storage servers. In Proc. of the 22nd Symposium on Reliable Distributed Systems, 2003.

[2] R. Bazzi and Y. Ding. Non-skipping timestamps for byzantine data storage systems. In Distributed Computing, 18th International Conference (DISC), pages 405-419, 2004.

[3] C. Cachin and S. Tessaro. Optimal resilience for erasurecoded byzantine distributed storage. Technical Report RZ 3575, IBM Research, Feb. 2005.

[4] D. K. Gifford. Weighted voting for replicated data. In Proc. of the Seventh Symposium on Operating Systems Principles, Dec. 1979.

[5] G. Goodson, J. Wylie, G. Ganger, and M. Reiter. Efficient byzantine-tolerant erasure-coded storage. In Proc. of the International Conference on Dependable Systems and Networks, June 2004.

[6] M. P. Herlihy and J. M. Wing. Axioms for Concurrent Objects. In Conference Record of the 14th Annual ACM Symposium on Principles of Programming Languages, 1987.

[7] IBM. http://www.ibm.com/security/cryptocards/, 2005.

[8] L. Lamport, R. Shostak, and M. Pease. The Byzantine Generals Problem. ACM Transactions on Programming Languages and Systems, 4(3):382-401, July 1982.

[9] D. Malkhi and M. Reiter. Byzantine Quorum Systems. Journal of Distributed Computing, 11(4):203-213, 1998.

[10] D. Malkhi and M. Reiter. Secure and scalable replication in phalanx. In Proc. of the 17th IEEE Symposium on Reliable Distributed Systems, Oct. 1998.

[11] D. Malkhi, M. Reiter, and N. Lynch. A Correctness Condition for Memory Shared by Byzantine Processes. Unpublished manuscript, Sept. 1998.

[12] J. Martin, L. Alvisi, and M. Dahlin. Minimal Byzantine storage. Technical Report TR-02-38, University of Texas at Austin, Department of Computer Sciences, Aug. 2002.

[13] R. H. Thomas. A majority consensus approach to concurrency control for multiple copy databases. ACM Transactions on Database Systems, 4(2):180-209, June 1979. 